\title{
Denervación renal para el tratamiento de la hipertensión arterial resistente en España. Registro Flex-Spyral
}

\section{RESUMEN (220 palabras)}

Introducción y objetivos: La denervación renal es una intervención percutánea para el tratamiento de la hipertensión arterial resistente. Estudios aleatorizados han mostrado resultados contradictorios sobre su eficacia. Se presentan los resultados de un registro de denervación en pacientes con hipertensión resistente según práctica clínica en nuestro país.

Métodos: Registro multicéntrico de pacientes consecutivos con hipertensión arterial resistente tratados con denervación renal en España en el período 2009-2018.

Resultados: Se han incluido 125 pacientes (edad media 56 \pm años, $41 \%$ sexo femenino, $14 \pm 9$ años de evolución de la hipertensión). La presión arterial sistólica y diastólica en la consulta y ambulatoria de 24 horas disminuyeron a los 6 meses de la intervención $(166 \pm 20 / 95 \pm 16$ a $149 \pm 22 / 87 \pm 16 \mathrm{mmHg}$ y $151 \pm 14 / 89 \pm 12$ a $143 \pm 15 / 84 \pm 11$ ambas con p <0,0001). A los 12 meses se mantuvo la reducción en la presión arterial con una

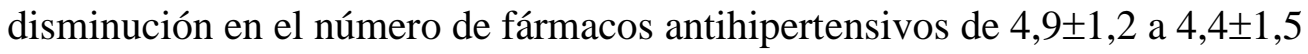
$(\mathrm{p}=0,0001)$. No hubo complicaciones importantes relacionadas con el procedimiento. La tasa de respuesta a la denervación al año fue del $80 \%$ si bien con una amplia variabilidad entre centros.

Conclusiones: La denervación renal en pacientes con hipertensión resistente se relacionó con una disminución de las cifras de presión arterial en la consulta y, lo que es más importante, en la monitorización ambulatoria de presión arterial, con una disminución significativa del tratamiento farmacológico.

\section{SUMMARY (209 words)}

Introduction and objectives: Renal denervation is a percutaneous intervention for the treatment of resistant hypertension. Randomized studies have shown contradictory results about its efficacy. We present the results of a renal denervation registry for the treatment of resistant hypertension in real life patients in our country.

Methods: Multicenter registry of consecutive patients with resistant arterial hypertension treated with renal denervation in Spain in the period 2009-2018. 
Results: 125 patients were included (mean age 56 years, $41 \%$ female sex, $14 \pm 9$ years of hypertension). Office systolic and diastolic blood pressure and ambulatory blood pressure monitoring decreased 6 months after the intervention $(166 \pm 20 / 96 \pm 16$ to $149 \pm 22 / 87 \pm 16 \mathrm{mmHg}$ and $151 \pm 14$ / 90 \pm 12 to $141 \pm 16 / 9012 \pm$ both with $\mathrm{p}<0.0001)$. At 12 months reduction in blood pressure was maintained with a decrease in the number of antihypertensive drugs from $4.9 \pm 1.2$ to $4.4 \pm 1.5(\mathrm{p}=0.0001)$. There were no significant complications related to the procedure. The rate of response to denervation at one year was $80 \%$, although with a wide variability between centers.

Conclusions: In patients with resistant hypertension, treatment with renal denervation was related to a decrease in office blood pressure and, more important, in ambulatory blood pressure monitoring, with a significant decrease in pharmacological treatment.

\section{Palabras Clave:}

Denervación renal percutánea, hipertensión arterial, sistema nervioso simpático, presión arterial

\section{$\underline{\text { Abreviaturas }}$}

DRP: denervación renal percutánea

HTA: hipertensión arterial

MAPA: monitorización ambulatoria de presión arterial

PA: presión arterial

4039 palabras 


\section{INTRODUCCIÓN}

La hipertensión arterial (HTA) es el factor de riesgo cardiovascular modificable con mayor prevalencia, afecta a más de mil millones de personas en todo el mundo y es responsable de la muerte de más de 9 millones de personas cada año (1). Estudios observacionales han mostrado una asociación gradual entre una mayor presión arterial (PA) y el desarrollo de enfermedad cardiovascular (2) de forma que un incremento de $20 \mathrm{mmHg}$ en la PA sistólica y $10 \mathrm{mmHg}$ en la PA diastólica duplican el riesgo de muerte cardiovascular (3).

A pesar de los esfuerzos para el control de la HTA, datos del año 2010 mostraron que, en países desarrollados, un tercio de los afectados desconocían tener la enfermedad, poco más de la mitad recibían tratamiento farmacológico y menos de un tercio presentaban un adecuado control tensional (4). Debido a las limitaciones del tratamiento farmacológico en la normalización de la PA en un número significativo de pacientes, en los últimos años han surgido distintas opciones no farmacológicas para el tratamiento de la HTA, como la denervación renal percutánea (DRP), tratamiento basado en la modulación del sistema nervioso simpático. El sistema nervioso simpático renal está involucrado en el desarrollo y la progresión de la HTA y en el daño orgánico mediado por la HTA. A nivel renal y ante distintos estímulos locales se genera una activación de las fibras nerviosas simpáticas aferentes hacia el sistema nervioso central; esta activación genera un incremento del tono simpático que produce un incremento en la PA al aumentar de la contractilidad cardíaca, promover la vasoconstricción, y estimular el eje renina-angiotensina-aldosterona y la retención renal de sodio, todo ello mediado por las vías simpáticas eferentes (5).

La DRP es un procedimiento mínimamente invasivo consistente en la introducción de un catéter en la arteria renal que mediante la liberación de energía (generalmente en forma de radiofrecuencia) produce una lesión en los nervios aferentes y eferentes del sistema nervioso simpático que discurren alrededor de las arterias renales (6). La interrupción de las vías simpáticas renales aferentes disminuye la presión arterial al disminuir el tono vascular y la contractilidad cardíaca a la vez que incrementa la natriuresis mientras que la interrupción de las vías simpáticas renales eferentes disminuye la activación del sistema renina-angiotensina-aldosterona y la retención de sodio (5).

A pesar de las expectativas iniciales generadas por los primeros estudios publicados en los que se mostró una mejoría de las cifras de presión arterial (PA) tras la DRP 
mediante radiofrecuencia en pacientes con HTA resistente $(7,8)$, la DRP no se mostró eficaz en mejorar la PA en comparación con un procedimiento simulado en el estudio Symplicity HTN-3 (9). Este resultado inesperado no se correspondía con los resultados en estudios previos $(7,8)$ ni con los datos reportados en registros de la vida real $(10)$ pero llevó prácticamente a un abandono de la técnica. Sin embargo, varios estudios publicados recientemente han demostrado, con un número limitado de pacientes con HTA moderada, que la DRP es eficaz para mejorar las cifras de PA en la consulta y, más importante, las cifras de PA en la monitorización ambulatoria de PA (MAPA) tanto en pacientes sin tratamiento farmacológico como en pacientes con tratamiento incompleto (11-13).

En nuestro país la DRP se inició de forma paralela a otros países europeos o Australia para el tratamiento de la HTA resistente, si bien su penetración fue significativamente menor. En el año 2014 se constituyó un grupo de trabajo en denervación renal con el objetivo de compartir experiencias en esta nueva técnica para el tratamiento de la HTA. El presente estudio presenta los datos de un registro multicéntrico retrospectivo sobre la DRP mediante catéter de radiofrecuencia unipolar (Symplicity Flex, Medtronic Inc.) o tetrapolar (Symplicity Spyral, Medtronic Inc.) para el tratamiento de la HTA resistente entre 2009 y 2018.

\section{MÉTODO}

Registro multicéntrico con recogida retrospectiva de datos en el que se han incluido pacientes con HTA resistente en los que se ha realizado DRP de forma consecutiva con catéter Symplicity Flex (2009-2015) o Spyral (2015-2018) en 7 centros españoles.

\section{Recogida de datos}

Los datos incluidos en el registro se han obtenido de forma retrospectiva a partir del análisis de las bases de datos locales en cada centro o bien de los historiales clínicos de los pacientes e incluyen datos clínicos basales y cifras de PA en consulta y en la MAPA antes del procedimiento y durante un seguimiento de 12 meses tras la DRP.

\section{Pacientes}

Se incluyeron pacientes con HTA resistente tratados de forma consecutiva mediante DRP. LA HTA resistente se definió como PA en consulta superior a 140/90 mmHg a 
pesar de tratamiento farmacológico estable durante un mínimo de 4 semanas con 3 o más fármacos antihipertensivos, uno de los cuales debía ser un diurético (13).

Todos los centros participantes en el registro tenían unidades especializadas en el tratamiento de la HTA y, por protocolo, se descartó causa secundaria de HTA resistente antes de la indicación de DRP mediante historia clínica, exploración física, test de imagen renal, cribado endocrinológico y polisomnografía. Los pacientes con causa secundaria conocida de HTA resistente no se consideraron candidatos a DRP. La realización de MAPA no se consideró necesaria para la indicación de DRP, pero se han registrado sus resultados cuando estaban disponibles. No se dispone de información sobre pacientes valorados para DRP en la que esta fue desestimada al considerarse el paciente no tributario.

\section{Mediciones de la presión arterial}

La PA en la consulta se determinó según las recomendaciones de las guías de práctica clínica de la Sociedad Europea de Cardiología y la Sociedad Europea de Hipertensión (14). La MAPA se realizó siguiendo las recomendaciones actuales y se evaluaron los valores promedio de la PA sistólica y de la PA diastólica durante las 24 horas (15).

\section{Procedimiento de denervación renal}

El procedimiento de DRP se realizó de acuerdo a las recomendaciones del fabricante de los dispositivos (6) por parte de cardiólogos intervencionistas, cirujanos vasculares o electrofisiólogos, según la práctica de cada centro. Entre los años 2009 y 2015 se realizó con el dispositivo monopolar Symplicity Flex (Medtronic Inc.) y a partir del año 2015 con el dispositivo tetrapolar Symplicity Spyral (Medtronic Inc.). Se consideró que un paciente era respondedor a la intervención cuando la PA sistólica en consulta a 12 meses disminuía un mínimo de $10 \mathrm{mmHg}$ o cuando la PA sistólica de 24 horas disminuía un mínimo de $5 \mathrm{mmHg}$.

\section{Análisis estadístico}

La comparación de las cifras de PA a lo largo del seguimiento se ha realizado mediante test $t$ de Student para datos pareados. Se han considerado como estadísticamente significativos los valores de $\mathrm{p} \leq 0,05$. Los análisis estadísticos se han realizado con Stata 15IC (Stata Corp, College Station, Texas, USA). 


\section{RESULTADOS}

\section{Población del estudio}

En el período del estudio se realizó DRP en 125 pacientes. Las características clínicas de los pacientes incluidos en el registro se resumen en la tabla 1.

\section{Procedimiento de denervación renal percutánea}

En los 125 pacientes se consiguió completar el procedimiento de DRP. No se recogieron datos sobre la anatomía de las arterias renales, presencia de arterias polares, o número o localización de las aplicaciones de radiofrecuencia. En todos los casos el procedimiento se realizó por acceso femoral. No se registraron complicaciones inmediatas relacionadas con la intervención a nivel renal y únicamente en dos casos se produjo una complicación relacionada con el punto de punción (2 casos de pseudoaneurisma femoral). No se detectó empeoramiento de la función renal relacionado con el procedimiento. La figura 1 muestra la distribución de los casos a lo largo de los años del registro; cabe destacar que los casos iniciales entre 2009 y 2011 fueron pacientes incluidos en los estudios Symplicity HTN 1 y Symplicity HTN 2. En 85 casos $(69 \%)$ se realizó el procedimiento con el catéter monopolar Symplicity Flex mientras que en los 38 restantes se realizó con el catéter Symplicity Spyral. La tasa global de respuesta a la DRP fue del 80,6\%; la respuesta fue del 79,6\% en los pacientes tratados con catéter Symplicity Flex y del $80 \%$ en los pacientes tratados con catéter Symplicity Spyral. Al analizar la tasa de respuesta por centros se observó una gran variabilidad, como se representa en la Figura 2.

\section{Cifras de PA en la consulta}

La determinación de PA en la consulta a lo largo del estudio se presentan en la tabla 2; la figura 3 representa el cambio en la PA en la consulta a lo largo del seguimiento. La PA sistólica y la PA diastólica disminuyeron a los 6 meses de la DRP $(166 \pm 20$ mmHg a $149 \pm 22$ y $95 \pm 16 \mathrm{mmHg}$ a $87 \pm 16 \mathrm{mmHg}$, respectivamente; en ambos casos con $\mathrm{p}<0,0001)$. La figura 4 representa la distribución de la PA sistólica en la consulta antes de la DRP y a los 6 y 12 meses de seguimiento.

\section{Cifras de PA en la MAPA}

La determinación de PA en la MAPA a lo largo del estudio se presentan en la tabla 3; la figura 5 representa el cambio en la PA en la MAPA a lo largo del seguimiento. La PA 


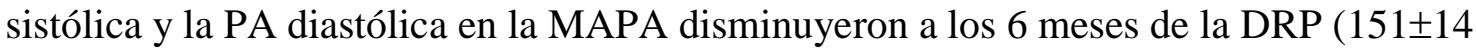
$\mathrm{mmHg}$ a $143 \pm 15$ y $89 \pm 12 \mathrm{mmHg}$ a $84 \pm 11$, respectivamente; en ambos casos con $\mathrm{p}<0,0001)$.

\section{Medicación antihipertensiva en relación a la respuesta a denervación}

La tabla 4 muestra el tratamiento farmacológico en el momento de la denervación y a los 12 meses de seguimiento. El número de fármacos antihipertensivos disminuyó de $4,9 \pm 1,2$ previo a la DRP a 4,4 $\pm 1,5$ a los 12 meses de seguimiento ( $\mathrm{p}=0,0001)$. La figura 6 muestra la relación entre los cambios en el tratamiento durante el seguimiento (incremento, descenso o sin cambios) y la respuesta a DRP (respuesta, no respuesta o imposibilidad de medir la respuesta al no disponer de datos de PA o MAPA). El único fármaco que incrementó su prescripción en el seguimiento fueron los antagonistas de la aldosterona. El 21\% de los 19 pacientes no respondedores estaban en tratamiento con antagonistas de la aldosterona antes de la denervación, frente al 55\% de los 76 pacientes respondedores. En el seguimiento, el tratamiento se incremento hasta el $37 \%$ en los no respondedores (retirada en un 1 paciente e inicio en 4) y hasta el $57 \%$ en los respondedores (retirada en 6 pacientes e inicio en 7 pacientes).

\section{DISCUSIÓN}

El análisis de los datos de este registro multicéntrico de pacientes con HTA resistente tratados con DRP en un contexto de la vida real ha mostrado que la técnica fue eficaz en conseguir una disminución de las cifras de PA en la consulta y, lo que es más importante, en la MAPA, con una disminución en el número de fármacos antihipertensivos. El procedimiento se mostró seguro, con una muy baja tasa de complicaciones relacionadas con la intervención. El éxito de la intervención fue independiente del tipo de catéter de radiofrecuencia utilizado, aunque se observó una variabilidad en la respuesta por centro.

La disminución en las cifras de PA que se muestra en nuestro registro está en concordancia a la que se había obtenido en estudios previos con datos de la vida real (10,16-19). Además, la mejoría en la PA se refleja también en la MAPA de 24 horas, hecho especialmente importante dado que un estudio reciente ha demostrado que es un factor predictor de mortalidad cardiovascular superior a las cifras de PA en la consulta 
(20). Además, esta mejoría se produjo junto con una disminución en el número de fármacos, a diferencia de lo observado en registros previos en que no hubo disminución del tratamiento farmacológico (10,16-19). El único fármaco que incrementó su prescripción fueron los antagonistas de la aldosterona. Estos fármacos se han erigido como fármaco de cuarta línea en pacientes con HTA resistente en las últimas guías europeas sobre el tratamiento de la HTA publicadas en 2018 con un nivel de recomendación IB (14). En este sentido, destaca que únicamente un 51\% de los candidatos a denervación estuvieran en tratamiento con estos fármacos, si bien se debe tener en cuenta que esta recomendación era más controvertida en las guías previas de 2013, en que tenía un nivel IIaB (21) y nuestra serie incluye pacientes desde 2009. Además, la respuesta a RDN fue significativamente peor en los pacientes que no tomaban antagonistas de la aldosterona. En la actualidad, los pacientes candidatos a DRP deberían estar en tratamiento con antagnositas de la aldosterona.

A pesar de los resultados prometedores de los estudios iniciales sobre DRP, el primer estudio en el que se comparó la DRP frente a un procedimiento de DRP simulado en pacientes con HTA resistente, el estudio SYMPLICITY HTN-3, no demostró beneficio del tratamiento sobre el control de la PA en la consulta ni en la MAPA (9). Los resultados de este estudio fueron cuestionados desde su publicación (22) y, en un análisis post-hoc, Kandzari et al observaron una respuesta menor a lo esperado en el grupo tratado con DRP y una respuesta muy por encima de lo esperado en el grupo control, en comparación con experiencias previas. Dicho análisis reveló potenciales factores de confusión que podrían explicar, al menos en parte, los inesperados resultados, como la inexperiencia de los operadores, la inclusión de pacientes con HTA sistólica aislada, pobre adherencia al tratamiento farmacológico antes de la selección y durante el estudio, inclusión de un alto porcentaje de pacientes de etnia afroamericana y deficiencias del procedimiento en términos de número y localización de las ablaciones (23). Respecto al procedimiento, surgieron dudas razonables sobre si la DRP se realizó de la misma manera que en los primeros estudios dado que la experiencia en el Registro GLOBAL SYMPLICITY, con operadores experimentados y en una población similar, demostró una caída media de la PAS de 19,3ะ22,4 a los 6 meses con un mayor número de ablaciones por paciente $(13,5 \pm 4,1)(10)$. La falta de ablaciones en los cuatro cuadrantes y el número de aplicaciones de radiofrecuencia se correlacionaron con la reducción de la PA (23). En nuestro registro no disponemos de información del 
procedimiento, pero si que llama la atención la heterogeneidad en la respuesta a DRP en distintos centros. Este hallazgo, congruente con lo observado en el estudio SYMPLICITY HTN-3, refleja muy probablemente la importancia de una buena selección de pacientes, así como de que el procedimiento se realice de la forma más exquisita posible. En este sentido, la indicación de DRP debería basarse siempre en los resultados de la MAPA para excluir pseudoresistencia o HTA de bata blanca, con exclusión de todas las causas secundarias de HTA resistente, con un ajuste de la medicación de acuerdo a las guías y con una medicación estable durante por lo menos 4-6 semanas antes de la denervación. Respecto a la intervención, una crítica que se realizó al SYMPLICITY HTN-3 fue que más del 50\% de pacientes incluidos fueron intervenidos por operadores que realizaban su primer o segundo procedimiento, de forma que la curva de aprendizaje podía influir en el resultado de la intervención (23). En nuestro registro los procedimientos fueron realizados por los mismos operadores (uno o dos por centro), con un volumen igual o superior a los estudios SYMPLICITY HTN 1 o 2 y muy superior al SYMPLICITY HTN 3. En la actualidad, la intervención debe buscar como objetivos el tratamiento de los 4 cuadrantes de la arteria renal, con el máximo número de aplicaciones de radiofrecuencia posibles (dejando una distancia de seguridad de $5 \mathrm{~mm}$ entre aplicaciones) y con el tratamiento del tronco y las ramas de la arteria renal (con un diámetro por encima de $3 \mathrm{~mm}$ ) (6). De forma inesperada, no se vio diferencia en la eficacia de la intervención cuando se comparó el catéter Symplicity Flex con el Symplicity Spyral, a pesar de que se trate de un catéter diseñado para mejorar la eficacia del procedimiento (6).

Los resultados de este registro refuerzan el papel de la DRP en el tratamiento de la HTA y, a pesar de que tras la publicación del SYMPLICITY HTN-3 la indicación de DRP se redujo drásticamente, los estudios SPYRAL HTN ON-MED, SPYRAL HTN OFFMED y RADIANCE SOLO, publicados recientemente, han proporcionado una primera evidencia, de forma consistente, sobre la posible utilidad clínica de la DRP en el tratamiento de pacientes que no pueden o no desean tomar fármacos antihipertensivos. Estos estudios incluyeron pacientes con HTA moderada, con tratamiento farmacológico no optimizado o sin tratamiento, y con un grupo control con simulación de DRP, demostrando una disminución significativa de la PA en consulta y en la MAPA en los pacientes tratados, si bien el número de pacientes incluidos es pequeño y el seguimiento es corto (11-13). Estos resultados deberán ser confirmados en estudios que ya están en 
marcha y probablemente cambien el enfoque del tratamiento de la HTA en los próximos años (24). En este sentido, nuestro registro muestra como gran novedad, respecto a previos, que con la DRP no solo es posible mejorar el control de la PA, sino que se puede hacer disminuyendo además el tratamiento farmacológico.

\section{Limitaciones}

Debido a la naturaleza observacional y retrospectiva de este estudio, los resultados presentados están sujetos a limitaciones. El criterio a la hora de indicar la intervención no fue estandarizado y podría variar entre los distintos centros. En algunos pacientes no están disponibles los registros de PA o de MAPA en el seguimiento y algunos de los pacientes incluidos aún no han completado 12 meses de seguimiento. La falta de datos del procedimiento como la anatomía de las arterias renales, presencia de arterias polares no tratadas, número de aplicaciones de radiofrecuencia, tratamiento solo de troncos principales o también de ramas de la arteria renal limita el análisis de posibles causas de no respuesta a DRP en un $20 \%$ de los casos incluidos, así como el análisis de la variabilidad de respuesta entre los distintos centros. La falta de un grupo control no permite establecer una relación clara y directa entre la DRP y la mejoría de las cifras de PA, que podría ser debida a efecto placebo, efecto Hawthorne o regresión a la media, que podrían llevar a una sobreestimación del efecto de la DRP (24), si bien los resultados son congruentes con estudios previos posteriores al SYMPLICITY HTN 3.

\section{CONCLUSIÓN}

En pacientes de la vida real con HTA resistente tratados con DRP por radiofrecuencia se observó una disminución de las cifras de PA en la consulta y, lo que es más importante, por MAPA de 24 horas, hasta en un $80 \%$ de los casos, con una disminución significativa del tratamiento farmacológico. El procedimiento se mostró seguro, con una muy baja tasa de complicaciones relacionadas con la intervención. La respuesta a DRP fue independiente del tipo de catéter de radiofrecuencia utilizado, aunque se observó una importante variabilidad en la respuesta por centro

\section{BIBLIOGRAFIA}

(1) World Health Organization. A global brief on hypertension: silent killer, global public health crisis. World Health Organization; 2013. [consultado 1 Sep 2018]. 
Disponible en http://www.who.int/cardiovascular diseases/publications/global brief hypertension/en/

(2) Rapsomaniki E, Timmis A, George J, et al. Blood pressure and incidence of twelve cardiovascular diseases: lifetime risks, healthy life-years lost, and agespecific associations in 1.25 million people. Lancet. 2014;383:1899-911

(3) Lewington S, Clarke R, Qizilbash N, et al. Age-specific relevance of usual blood pressure to vascular mortality: a meta-analysis of individual data for one million adults in 61 prospective studies. Lancet. 2002;360:1903-13

(4) Mills KT, Bundy JD, Kelly TN, et al. Global disparities of hypertension prevalence and control. A systematic analysis of population-based studies from 90 countries. Circulation 2016; 134:441-450

(5) DiBona GF, Esler M. Translational medicine: the antihypertensive effect of renal denervation . Am J Physiol Regul Integr Comp Physiol 2010;298:R245R253

(6) Rodríguez-Leor O, Cid-Alvarez B, Moreno R, Pérez de Prado A. Selección de lo mejor del año 2018 en denervación simpática renal en el tratamiento de la $\begin{array}{llll}\text { hipertensión } & \text { arterial. } & \text { REC } & \text { CardioClinics }\end{array}$ https://doi.org/10.1016/j.rccl.2019.01.009

(7) Krum H, Schlaich M, Whitbourn R, et al. Catheter-based renal sympathetic denervation for resistant hypertension: a multicentre safety and proof-ofprinciple cohort study. Lancet 2009; 373:1275-1281

(8) Esler MD, Krum H, Schlaich M, et al. Renal sympathetic denervation in patients with treatment-resistant hypertension (The Symplicity HTN-2 Trial): a randomised controlled trial. Lancet 2010;376:1903-1909Symplicity 3

(9) Bhatt DL, Kandzari DE, O'Neill WW, et al. A controlled trial of renal denervation for resistant hypertension. N Engl J Med 2014; 370:1393-1401

(10) Böhm M, Mahfoud F, Ukena C, et al. First report of the Global SYMPLICITY Registry on the effect of renal artery denervation in patients with uncontrolled hypertension Hypertension 2015;65:766-774

(11) Townsend RR, Mahfoud F, Kandzari DE, et al. Catheter-based renal denervation in patients with uncontrolled hypertension in the absence of antihypertensive medications (SPYRAL HTN-OFF MED): a randomised, shamcontrolled, proof-of-concept trial. Lancet 2017;390:2160-70 
(12) Kandzari DE, Böhm M, Mahfoud F, et al. Effect of renal denervation on blood pressure in the presence of antihypertensive drugs: 6-month efficacy and safety results from the SPYRAL HTN-ON MED proof-of-concept randomised trial. Lancet. 2018;391:2346-55

(13) Azizi M, Schmieder RE, Mahfoud F, et al. Endovascular ultrasound renal denervation to treat hypertension (RADIANCE-HTN SOLO): a multicentre, international, single-blind, randomised, sham-controlled trial. Lancet 2018;391:2335-45

(14) Williams B, Mancia G, Spiering W, Rosei EA, et al. 2018 ESC/ESH guidelines for the management of arterial hypertension. Rev Esp Cardiol 2019;72:160

(15) O’Brien E, Parati G, Stergiou G, Asmar R, Beilin L, Bilo G, et al. European Society of Hypertension position paper on ambulatory blood pressure monitoring. J Hypertens 2013; 31:1731-1768

(16) Sharp AS, Davies JE, Lobo MD, Bent CL, et al. Renal artery sympathetic denervation: observations from the UK experience. Clin Res Cardiol 2016; 105:544-52.

(17) Zweiker D, Lambert T, Steinwender C, Weber T, et al. Effects of renal denervation documented in the Austrian National Multicentre Renal Denervation Registry. PLoS One 2016; 11:e0161250.

(18) Tsioufis C, Ziakas A, Dimitriadis K, Davlouros P, et al. Blood pressure response to catheter-based renal sympa- thetic denervation in severe resistant hypertension: data from the Greek Renal Denervation Registry. Clin Res Cardiol 2017; 106:322-330

(19) Völz S, Spaak J, Elf J, Jägrén C, et al. Renal sympathetic denervation in Sweden: a report from the Swedish registry for renal denervation. J Hypertens 2018;36:151-158

(20) Banegas JR, Ruilope LM, de la Sierra A, Vinyoles E, et al. Relationship between clinic and ambulatory blood-pressure measurements and mortality. $\mathrm{H}$ Engl J Med 2018;378:1509-1520

(21) Mancia G, Fagard R, Narkiewicz K, et al. 2013 ESH/ESC guidelines for the management of arterial hypertension. Eur Heart J 2013;34:2159-2219

(22) Rodriguez-Leor O, Bonet J, Bayes-Genis A. Renal denervation for resistant hypertension. N Engl J Med 2014;371:182-3 
(23) Kandzari DE, Bhatt DL, Brar S, et al. Predictors of blood pressure response in the SYMPLICITY HTN-3 trial. Eur Heart J 2015;36: 219-27

(24) Mahfoud F, Schlaich M, Böhm M, Esler M, Lüscher TF. Catheter-based renal denervation: the next chapter begins. Eur Heart J 2018;39:4144-4149

(25) Pocock SJ, Bakris G, Bhatt DL, Brar S, et al. Regression to the mean in SYMPLICITY HTN-3: implications for design and reporting of future trials. J Am Coll Cardiol 2016; 68:2016 - 2025 


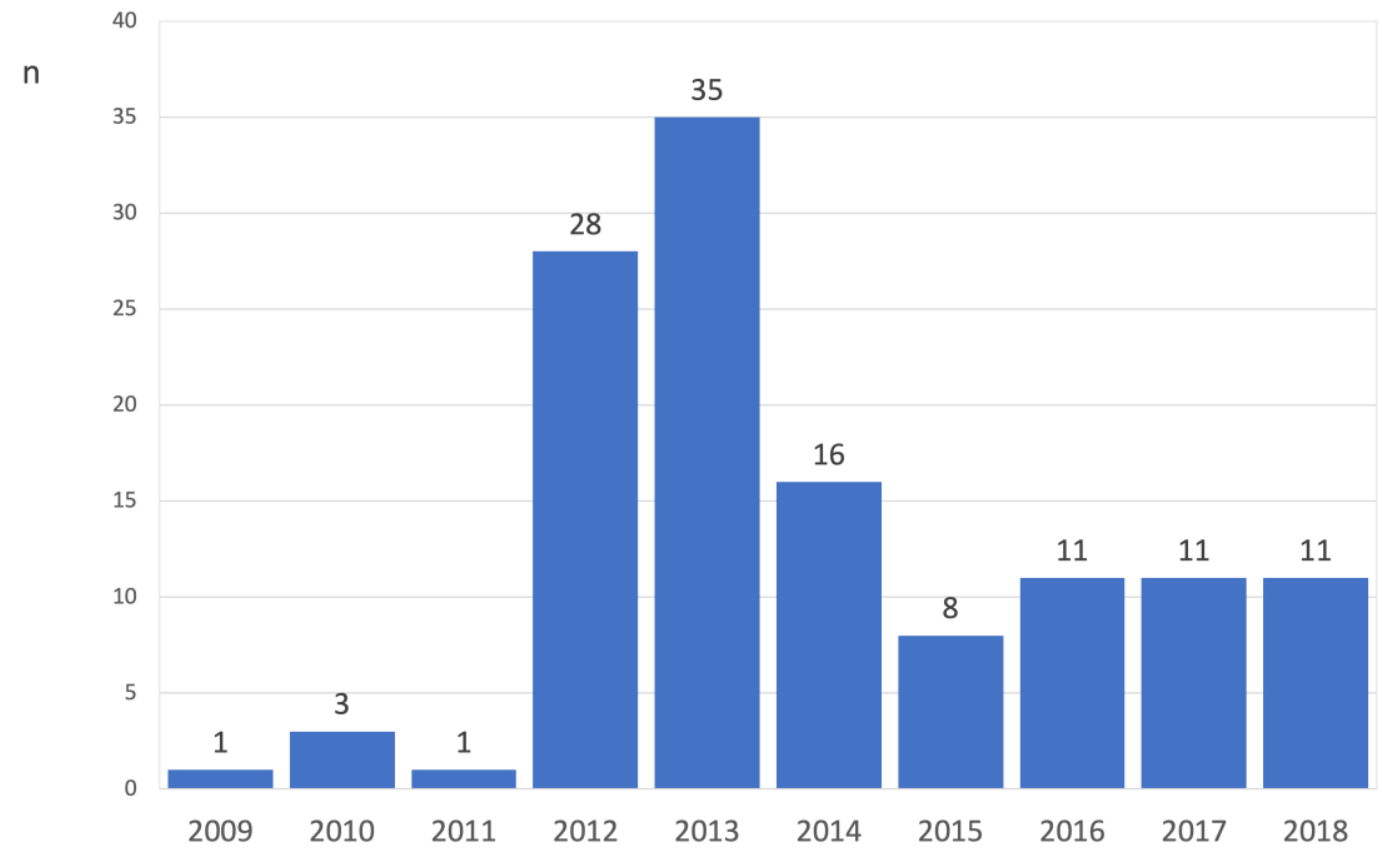

Figura 1. Distribución temporal de los casos a lo largo de los años del registro. Los casos incluidos entre 2009 y 2011 formaron parte de los estudios Symplicity HTN 1 y 2.

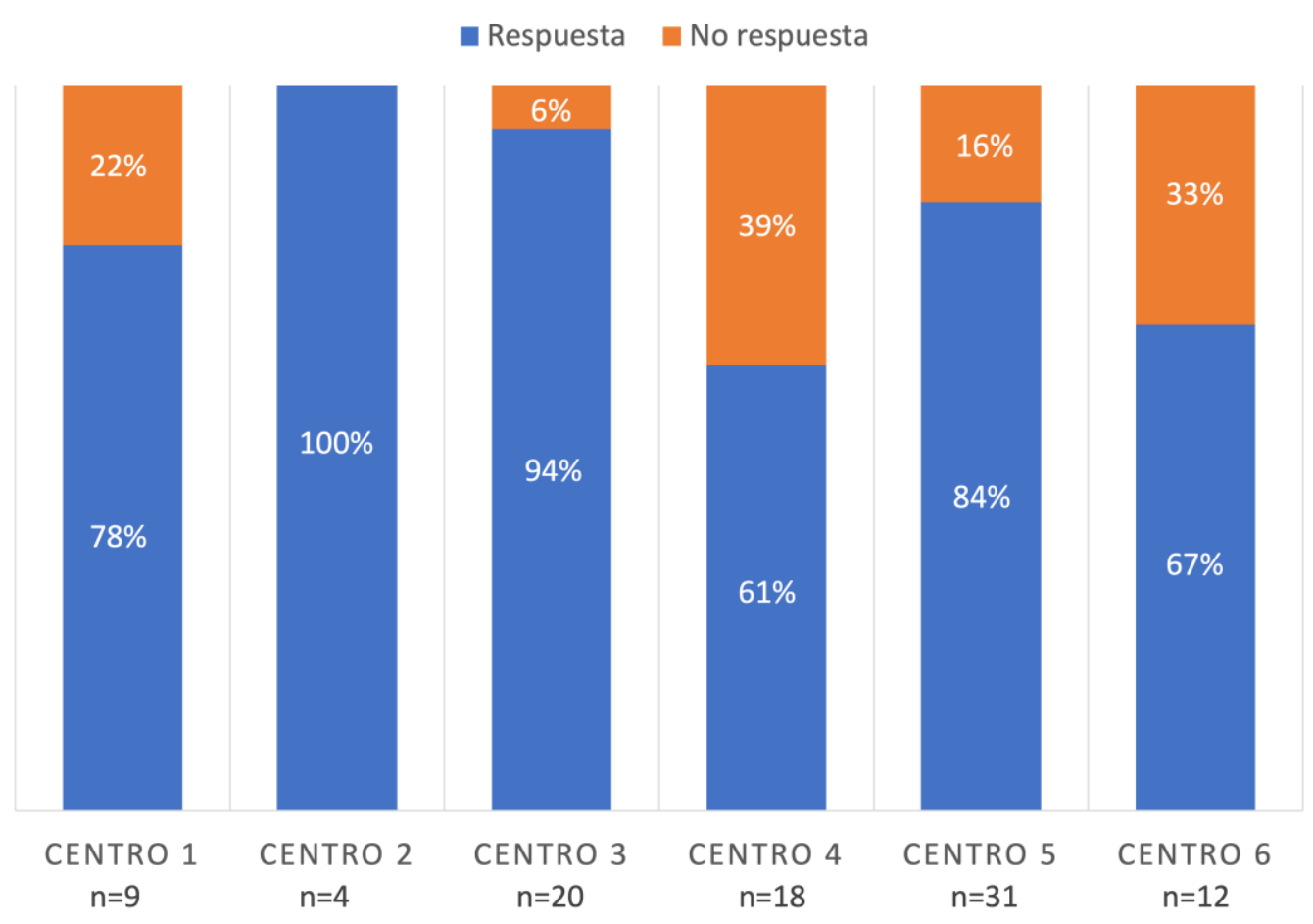

Figura 2. Respuesta a la DRP por centros. Los datos de un centro no se han incluido al no disponer de seguimiento a 12 meses de los pacientes tratados con DRP. 


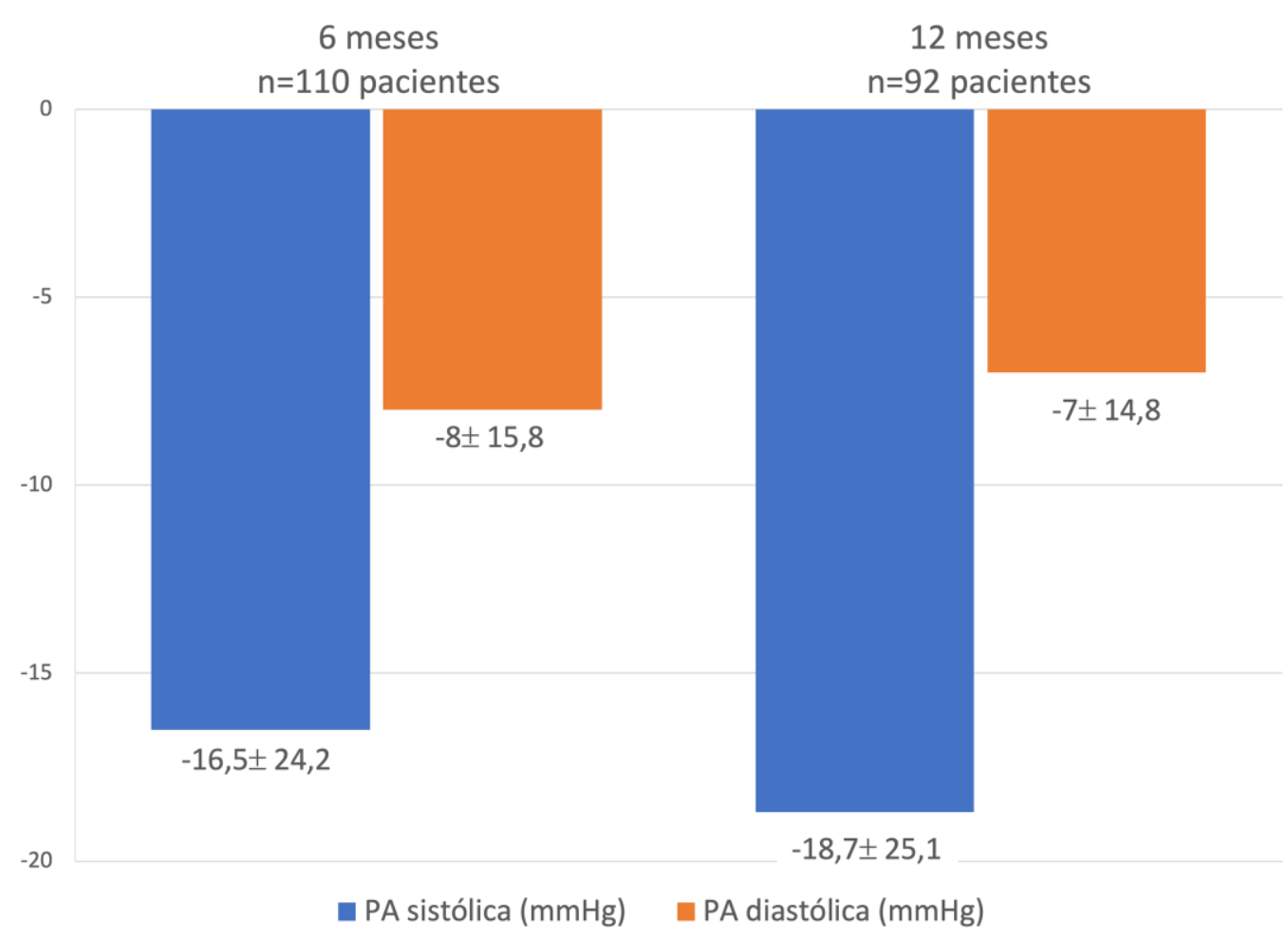

Figura 3. Cambio en la PA en la consulta a lo largo del seguimiento.

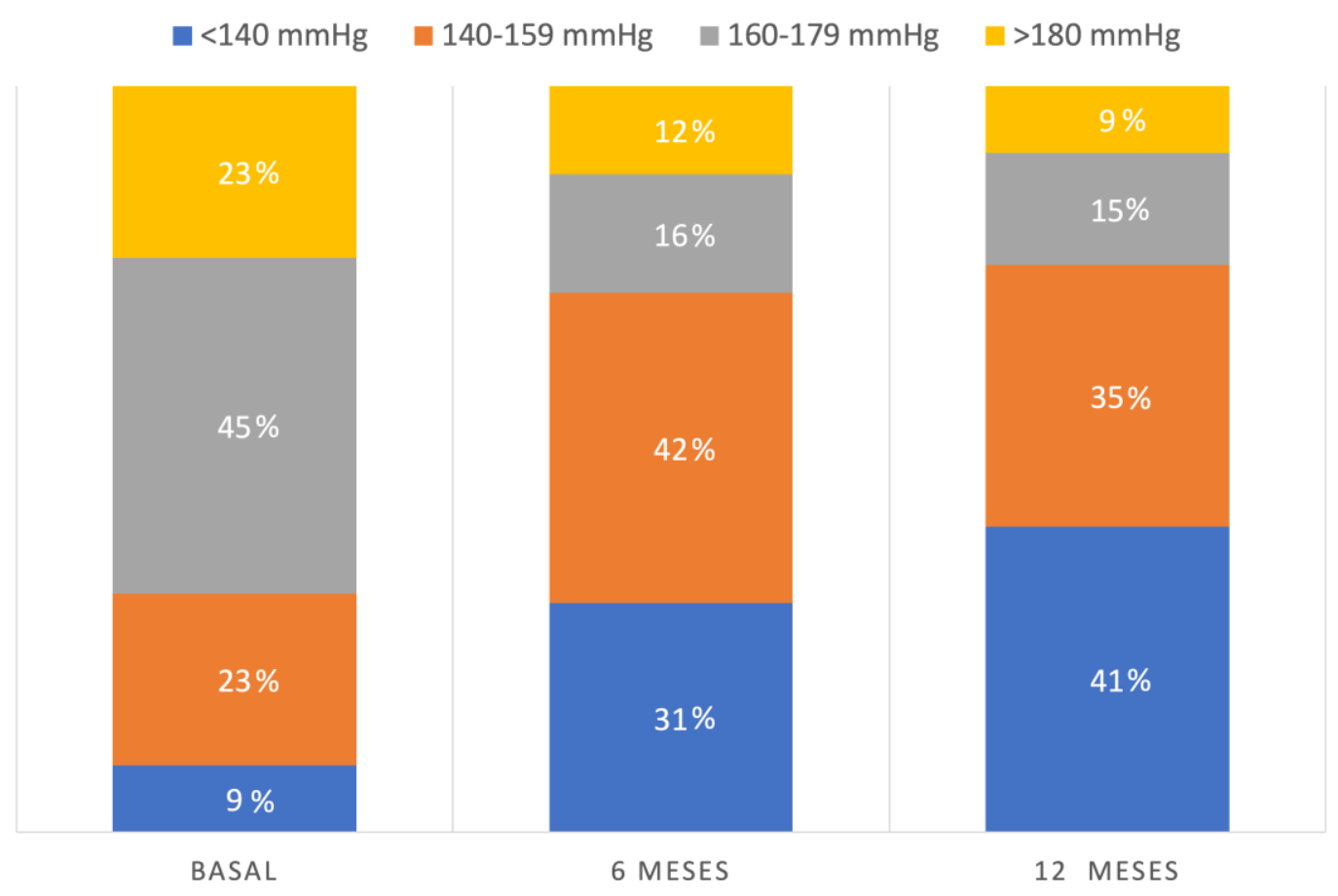

Figura 4. Distribución de la PA sistólica en la consulta antes de la DRP y a los 6 y 12 meses de seguimiento. 


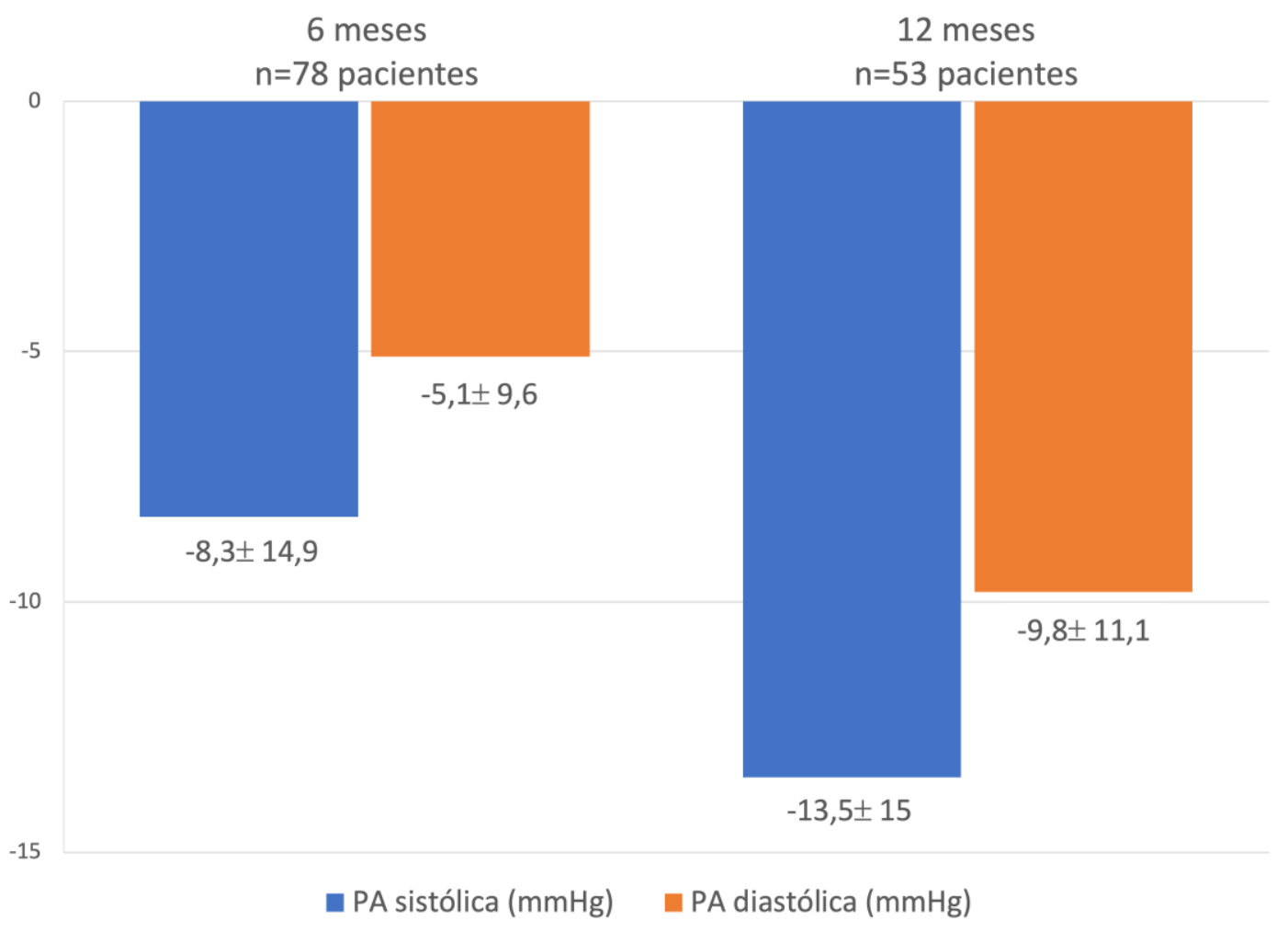

Figura 5. Cambio en la PA en la MAPA a lo largo del seguimiento

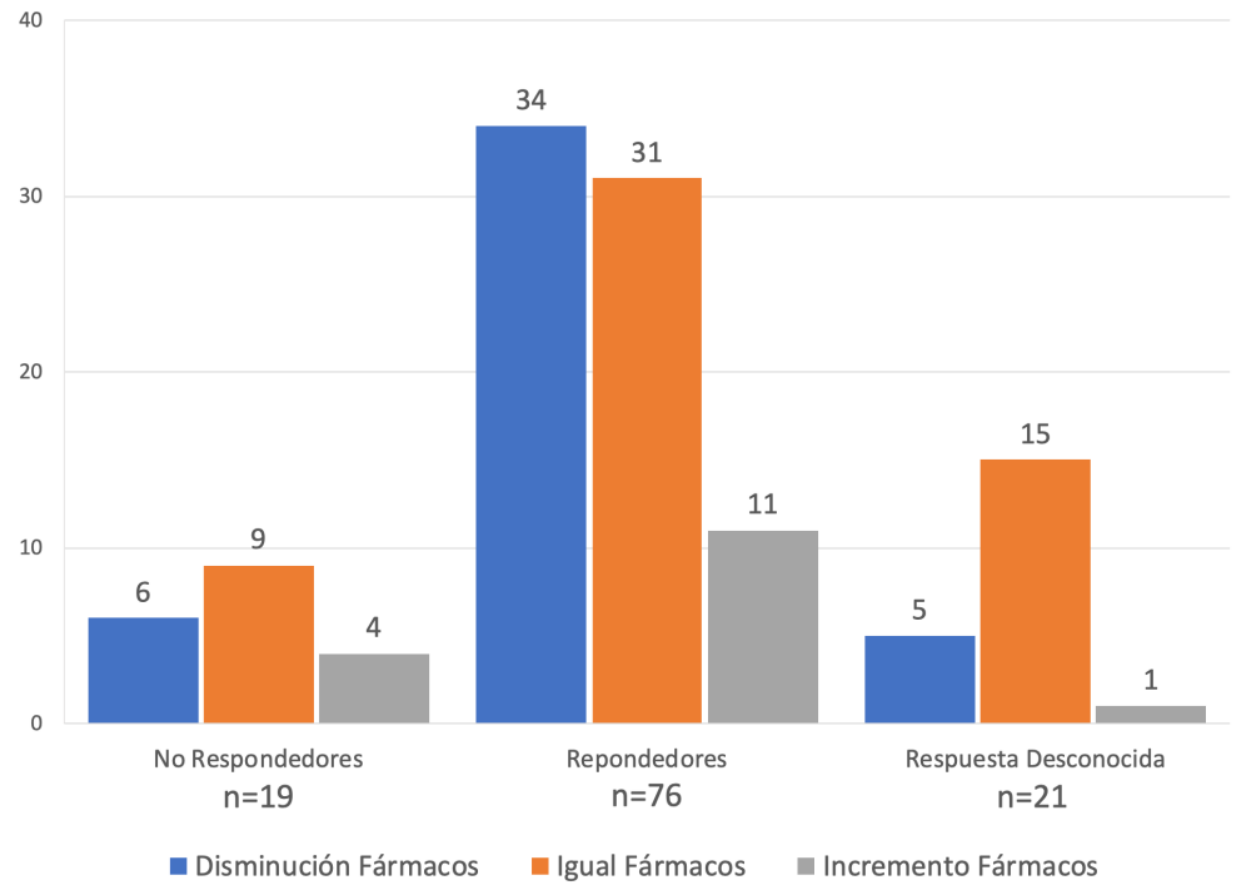

Figure 6. Relationship between the changes in treatment during follow-up (increase, decrease, or no change) and the response to pRDN (response, without 
response, or impossible to measure due to a lack of BP or ABPM data). ABPM, 24-hour ambulatory blood pressure monitoring; BP, blood pressure; $\mathrm{pRDN}$, percutaneous renal denervation. 


\section{PUNTOS CLAVE:}

\section{¿Qué se sabe del tema?}

- La denervación renal es una intervención para el tratamiento de la hipertensión arterial resistente que ha mostrado resultados contradictorios en términos de eficacia.

- Recientemente se han publicado tres estudios aleatorizados con un número pequeño de pacientes y con seguimiento a corto plazo en el que la denervación se mostró eficaz en el control de la hipertensión arterial moderada sin tratamiento farmacológico o con tratamiento incompleto.

\section{¿Qué aporta de nuevo?}

- Este trabajo muestra los resultados de la denervación en pacientes de la vida real con hipertensión arterial resistente en nuestro medio, con una mejoría significativa tanto en la presión arterial en la consulta como en la monitorización ambulatoria.

- La mejoría en las cifras de presión arterial se acompaña además de una disminución del número de fármacos antihipertensivos.

- Se ha detectado una gran variabilidad entre centros en la respuesta a la denervación renal, hecho que pone de manifiesto la importancia de una adecuada selección de los pacientes y que la intervención se realice de la forma más exquisita posible. 
Tabla 1. Características clínicas basales de los 125 pacientes con HTA resistente tratados con DRP

\begin{tabular}{|l|r|}
\hline Edad (años) & $56 \pm 13$ \\
\hline Sexo femenino, n(\%) & $51(41)$ \\
\hline Tiempo desde el diágnóstico de HTA (años) & $14 \pm 9$ \\
\hline IMC (kg/m2) & $31 \pm 6$ \\
\hline Tabaquismo activo, n(\%) & $37(30)$ \\
\hline Diabetes mellitus, n(\%) & $38(31)$ \\
\hline Dislipemia, n(\%) & $79(64)$ \\
\hline Apnea obstructiva del sueño, n(\%) & $38(31)$ \\
\hline Insuficiencia cardíaca previa, n(\%) & $15(3)$ \\
\hline Cardiopatía isquémica previa, n(\%) & $11(9)$ \\
\hline Accidente cerebrovascular previo, n(\%) & $6(5)$ \\
\hline Fibrilación auricular previa conocida, n(\%) & $32(26)$ \\
\hline Comorbilidad cardiovascular, n(\%) & $15(\%)$ \\
\hline
\end{tabular}

IMC: índice de masa corporal. Se ha considerado comorbilidad cardiovascular cuando el paciente presentaba, por lo menos, una de las siguientes: insuficiencia cardíaca, cardiopatía isquémica, accidente cerebrovascular o fibrilación auricular

Tabla 2. Evolución de la PA en la consulta

\begin{tabular}{|l|c|c|c|c|c|c|}
\hline & $\begin{array}{c}\text { Basal } \\
\mathrm{n}=110\end{array}$ & $\begin{array}{c}\text { 6 meses } \\
\mathrm{n}=110\end{array}$ & $\mathbf{p}$ & $\begin{array}{c}\text { Basal } \\
\mathrm{n}=92\end{array}$ & $\begin{array}{c}\text { 12 meses } \\
\mathrm{n}=92\end{array}$ & $\mathbf{p}$ \\
\hline $\begin{array}{l}\text { PA sistólica } \\
\text { en consulta } \\
(\mathbf{m m H g})\end{array}$ & $\begin{array}{c}166 \pm 20 \\
\mathrm{IC} 95 \%(162-170)\end{array}$ & $\begin{array}{c}149 \pm 22 \\
\mathrm{IC} 95 \%(145-154)\end{array}$ & $<0,0001$ & $\begin{array}{c}165 \pm 20 \\
\mathrm{IC} 95 \%(160-169)\end{array}$ & $\begin{array}{c}146 \pm 22 \\
\text { IC95\% (141-151) }\end{array}$ & $<0,0001$ \\
\hline $\begin{array}{l}\text { PA diastólica } \\
\text { en consulta } \\
\text { (mmHg) }\end{array}$ & $\begin{array}{c}95 \pm 16 \\
\mathrm{IC} 95 \%(92-98)\end{array}$ & $\begin{array}{c}87 \pm 16 \\
\mathrm{IC} 95 \%(84-90)\end{array}$ & $<0,001$ & $\begin{array}{c}94 \pm 16 \\
\text { IC95\% (91-97) }\end{array}$ & $\begin{array}{c}87 \pm 16 \\
\text { IC95\% (84-90) }\end{array}$ & $<0,0001$ \\
\hline
\end{tabular}

Análisis de la evolución de la PA en consulta en los 108 pacientes con control a 6 meses y en los 98 pacientes con control a 12 meses. La PA se expresa como media \pm desviación estándar y con el intervalo de confianza del 95\%. P respecto a cifras basales. 
Tabla 3. Evolución de la PA en la MAPA

\begin{tabular}{|c|c|c|c|c|c|c|}
\hline & $\begin{array}{l}\text { Basal } \\
n=68\end{array}$ & $\begin{array}{c}6 \text { meses } \\
n=68\end{array}$ & $\mathbf{p}$ & $\begin{array}{l}\text { Basal } \\
n=53\end{array}$ & $\begin{array}{c}12 \text { meses } \\
n=53\end{array}$ & $\mathbf{p}$ \\
\hline $\begin{array}{l}\text { PA sistólica en } \\
\text { MAPA (mmHg) }\end{array}$ & $\begin{array}{c}151 \pm 14 \\
\text { IC95\% (148-154) }\end{array}$ & $\begin{array}{c}143 \pm 15 \\
\text { IC95\% (148-154) }\end{array}$ & $<0,0001$ & $\begin{array}{c}150 \pm 14 \\
\text { IC95\% (146-154) }\end{array}$ & $\begin{array}{c}136 \pm 16 \\
\text { IC95\% (132-141) }\end{array}$ & $<0,0001$ \\
\hline $\begin{array}{l}\text { PA diastólica en } \\
\text { MAPA (mmHg) }\end{array}$ & $\begin{array}{c}89 \pm 12 \\
\text { IC } 95 \%(86-92)\end{array}$ & $\begin{array}{c}84 \pm 11 \\
\text { IC95\% (82-87) }\end{array}$ & $<0,0001$ & $\begin{array}{c}92 \pm 12 \\
\text { IC95\% (89-95) }\end{array}$ & $\begin{array}{c}82 \pm 14 \\
\text { IC95\% (78-86) }\end{array}$ & $<0,0001$ \\
\hline
\end{tabular}

Análisis de la evolución de la PA en la MAPA en los 68 pacientes con control a 6 meses y en los 53 pacientes con control a 12 meses. La PA se expresa como media \pm desviación estándar y con el intervalo de confianza del $95 \%$. P respecto a cifras basales.

Tabla 4. Evolución del tratamiento farmacológico a lo largo del estudio

\begin{tabular}{|l|c|c|}
\hline & $\begin{array}{c}\text { Basal } \\
\mathrm{n}=125\end{array}$ & $\begin{array}{c}12 \text { meses } \\
\mathrm{n}=114\end{array}$ \\
\hline IECA & $29(24 \%)$ & $24(21 \%)$ \\
\hline ARA II & $102(84 \%)$ & $87(76 \%)$ \\
\hline Beta bloqueante & $82(67 \%)$ & $75(66 \%)$ \\
\hline Antagonista del calcio & $94(76 \%)$ & $84(74 \%)$ \\
\hline Diurético de asa & $39(32 \%)$ & $27(24 \%)$ \\
\hline Tiacida & $84(68 \%)$ & $70(61 \%)$ \\
\hline Antagonista de la aldosterona & $63(51 \%)$ & $66(58 \%)$ \\
\hline Alfa bloqueante & $56(46 \%)$ & $42(37 \%)$ \\
\hline Otros & $37(30 \%)$ & $24(21 \%)$ \\
\hline
\end{tabular}

IECA (inhibidor del enzima conversor de la Angiotensina); ARA II (antagonista del receptor de la angiotensina II) 